\title{
An antidote to persistence
}

Toxin-antitoxin modules that are composed of a stable toxin and a labile antitoxin can regulate bacterial growth, with growth toxicity mediated by the toxin and countered by the antitoxin. Of 14 putative toxin-antitoxin modules that have been identified in the genome of the enteric pathogen Salmonella enterica subsp. enterica serovar Typhimurium, three are predicted to encode an $N$-acetyltransferase as a new class of toxin. Now, Cheverton et al. show that one of these toxins, TacT, acetylates aminoacyl-tRNAs to inhibit translation, slow growth and increase persister formation, and that TacT toxicity can be reversed by an antitoxin-independent mechanism that enables $S$. Typhimurium to resume growth.

Growth assays of $S$. Typhimurium mutants that overexpressed TacT and/or its antitoxin confirmed that they form a bona fide toxin-antitoxin module. Furthermore, in vitro acetylation assays and cell-free expression assays showed that TacT acetylates the amino acid of aminoacyl-tRNAs

S. Typhimurium uses an

antitoxinindependent detoxification mechanism to resume growth following TacT toxicity

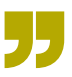

TacT did not impair growth at exponential phase but instead delayed the exit from lag phase by several hours. Infections with $S$. Typhimurium are often characterized by persistence, in which transiently slow or non-growing cells, such as those in lag phase, survive antibiotic treatment. Consistent with a role for TacT in persister formation, expression of the toxin increased the number of persister cells when $S$. Typhimurium was exposed to bactericidal concentrations of three different classes of antibiotic in vitro.

These growth and persister formation phenotypes could be prevented by expression of the antitoxin; however, as TacT inhibits translation, $S$. Typhimurium is probably not able to produce the antitoxin once TacT toxicity has occurred. To see whether alternative mechanisms might instead be responsible for detoxification, the authors designed a screen for genes that suppress TacT toxicity, which identified peptidyl-tRNA hydrolase (Pth). The ability of Pth to deacetylate acetylated aminoacyl-tRNAs, suppress TacT-induced growth defects and prevent the increase in persister formation that is associated with TacT toxicity was confirmed in vitro by acetylation assays, growth assays and antibiotic-exposure assays, respectively. Furthermore, the identification of Pth as a detoxifying factor suggested that TacT acetylates the primary amine of aminoacyl-tRNAs, which molecular modelling indicated is located in close proximity to acetylCoA in the TacT active site. Finally,

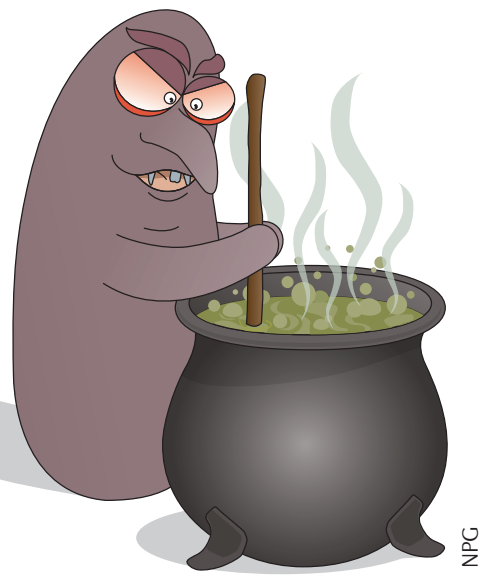

CobB, which is the only non-specific deacetylase known to be encoded by the $S$. Typhimurium genome, may further contribute to detoxification, as deletion of $c o b B$ enhanced the extension of the lag phase produced by TacT and even resulted in a new phenotype of slow growth during exponential phase. However, whether CobB directly deacetylates aminoacyl-tRNAs has not yet been determined.

Together, the findings of the study show that translational inhibition, and ultimately persister formation, mediated by TacT depends not only on the relative abundances of the toxin and antitoxin, but also on the balance of acetylation by TacT and deacetylation by Pth (and possibly CobB), which determines the acetylation state of aminoacyl-tRNAs. Thus, $S$. Typhimurium uses an antitoxin-independent detoxification mechanism to resume growth following TacT toxicity.

Naomi Attar

ORIGINAL ARTICLE Cheverton, A. M. et al. A Salmonella toxin promotes persister formation through acetylation of tRNA. Mol. Cell http:// dx.doi.org/10.1016/j.molcel.2016.05.002 (2016) 